\title{
A note on a construction of J. F. Feinstein
}

by

\author{
M. J. HeATh (Nottingham)
}

\begin{abstract}
In [6] J. F. Feinstein constructed a compact plane set $X$ such that $R(X)$, the uniform closure of the algebra of rational functions with poles off $X$, has no non-zero, bounded point derivations but is not weakly amenable. In the same paper he gave an example of a separable uniform algebra $A$ such that every point in the character space of $A$ is a peak point but $A$ is not weakly amenable. We show that it is possible to modify the construction in order to produce examples which are also regular.
\end{abstract}

1. Introduction. A uniform algebra $A$ on a compact (Hausdorff) space $X$ is said to be regular on $X$ if for any point $x$ in $X$ and any compact subset $K$ of $X \backslash\{x\}$ there is a function $f$ in $A$ such that $f(x)=1$ and $f$ is zero on $K$. We call $A$ regular if it is regular on its character space, and trivial if it is $C(X)$, the uniform algebra of all continuous functions on $X$. The first example of a non-trivial regular uniform algebra was given by McKissick [8] (see also [7]; [11, Chapter 37]): the example was $R(X)$ for a compact plane set $X$.

The notion of weak amenability was introduced in [1]. A commutative Banach algebra $A$ is said to be weakly amenable if there are no non-zero, continuous derivations from $A$ into any commutative Banach $A$-bimodule. It is proved in [1] that this is equivalent to there being no non-zero continuous derivations into the dual module $A^{\prime}$.

As point derivations may be regarded as derivations into 1-dimensional, commutative Banach modules it is a necessary condition for weak amenability that there be no non-zero, bounded point derivations. However, this condition is not sufficient, even for uniform algebras: in [6] J. F. Feinstein constructed a compact plane set $X$ such that the uniform algebra $R(X)$ has no non-zero, bounded point derivations but $R(X)$ is not weakly amenable. It was not clear whether such an example could also be regular. In this note we show that it can.

Notation. Throughout this paper $Q$ will refer to the compact plane set $\{x+i y: x, y \in[-1,1]\}$.

2000 Mathematics Subject Classification: Primary 46J10; Secondary 46H25. 
For a plane set $X$ and a function $f \in C(X),|f|_{X}$ will be the uniform norm of $f$ on $X, \sup \{|f(z)|: z \in X\}$. For a compact plane set $X$ we denote by $R_{0}(x)$ the set of restrictions to $X$ of rational functions with poles off $X$. Hence the uniform algebra $R(X)$ is the uniform closure of $R_{0}(X)$ in $C(X)$.

If $D$ is a disc in the plane then $r(D)$ shall refer to its radius.

Let $\mu$ be a complex measure on a compact plane set $X$ such that the bilinear functional on $R_{0}(X) \times R_{0}(X)$ defined by

$$
(f, g) \mapsto \int_{X} f^{\prime}(x) g(x) d \mu(x)
$$

is bounded. Then, as in [6], we may extend by continuity to $R(X) \times R(X)$ and obtain a continuous derivation $D$ from $R(X)$ to $R(X)^{\prime}$ such that for any $f$ and $g$ in $R_{0}(X)$ we have

$$
D(f)(g)=\int_{X} f^{\prime}(x) g(x) d \mu(x) .
$$

In the next section, we strengthen Körner's [7] version of McKissick's Lemma to allow greater control over the centres and radii of the discs removed. Using this we modify Feinstein's [6] construction so that $R(X)$ is regular. In fact we prove the following theorem.

TheOREM 1.1. For each $C>0$ there is a compact plane set $X$ obtained by deleting from $Q$ a countable union of Jordan domains such that $\partial Q$ is a subset of $X, R(X)$ is regular and has no non-zero, bounded point derivations and, for all $f, g$ in $R_{0}(X)$,

$$
\left|\int_{\partial Q} f^{\prime}(z) g(z) d z\right| \leq C|f|_{X}|g|_{X}
$$

If we let $X$ be a compact plane set constructed as in Theorem 1.1 we have, by the discussion above, a non-zero continuous derivation $D$ from $R(X)$ to $R(X)^{\prime}$ such that

$$
D(f)(g)=\int_{\partial Q} f^{\prime}(z) g(z) d z
$$

for $f, g \in R_{0}(X)$. So $R(X)$ is not weakly amenable.

2. The construction. Our main new tool will be the following theorem, which is a variation on McKissick's result in [8] (see also [7]).

TheOREm 2.1. For any $C_{0}>0$ there is a compact plane set $X_{1}$ obtained by deleting from $Q$ a countable union of open discs $\left(D_{n}\right)_{n=1}^{\infty}$ whose closures are in $\operatorname{int}(Q)$ such that $R\left(X_{1}\right)$ is regular and, letting $s_{n}$ be the distance 
from $D_{n}$ to $\partial Q$,

$$
\sum_{n=1}^{\infty} \frac{r\left(D_{n}\right)}{s_{n}^{2}}<C_{0} .
$$

In order to prove Theorem 2.1 we require a series of lemmas which are variations on the results of [7].

The following is [7, Lemma 2.1].

Lemma 2.2. If $N \geq 2$ is an integer and $h_{N}(z)=1 /\left(1-z^{N}\right)$ then the following hold:

(i) $\left|h_{N}(z)\right| \leq 2|z|^{-N}$ for $|z|^{N} \geq 2$;

(ii) $\left|1-h_{N}(z)\right| \leq 2|z|^{N}$ for $|z|^{N} \leq 2^{-1}$;

(iii) $h_{N}(z) \neq 0$ for all $z$.

Further if $(8 \log N)^{-1}>\delta>0$ then:

(iv) $\left|h_{N}(z)\right| \leq 2 \delta^{-1}$ provided only that $|z-w| \geq \delta N^{-1}$ whenever $w^{N}=1$.

The following is a variant of [7, Lemma 2.2].

Lemma 2.3. If in Lemma 2.2 we set $N=n 2^{2 n}$ with $n$ sufficiently large then:

(i) $\left|h_{N}(z)\right| \leq(n+1)^{-4}$ for $|z| \geq 1+2^{-(2 n+1)}$;

(ii) $\left|1-h_{N}(z)\right| \leq(n+1)^{-4}$ for $|z| \leq 1-2^{-(2 n+1)}$;

(iii) $h_{N} \neq 0$ for all $z$;

(iv) $\left|h_{N}(z)\right| \leq n^{-4} 2^{2 n+1}$ provided only that $|z-w| \geq n^{-5} 2^{-4 n}$ whenever $w^{N}=1$.

Proof. Parts (i), (ii) and (iii) are the corresponding parts of [7, 2.2]. Part (iv) follows on putting $\delta=n^{-3} 2^{-2 n}$.

From this we obtain the following variation on [7, Lemma 2.3].

Lemma 2.4. Provided only that $n$ is sufficiently large we can find a finite collection $A(n)$ of disjoint open discs and a rational function $g_{n}$ such that, letting $s_{0}(\Delta)=\operatorname{dist}(\Delta, \mathbb{R} \cup i \mathbb{R})$ for a disc $\Delta$, the following hold:

(i) $\sum_{\Delta \in A(n)} r(\Delta) / s_{0}(\Delta)^{2}<n^{-2}$ and so $\sum_{\Delta \in A(n)} r(\Delta) \leq n^{-2}$;

(ii) the poles of $g_{n}$ lie in $\bigcup_{\Delta \in A(n)} \Delta$;

(iii) $\left|g_{n}(z)\right| \leq(n+1)^{-4}$ for $|z| \geq 1-2^{-(2 n+1)}$;

(iv) $\left|1-h_{N}(z)\right| \leq(n+1)^{-4}$ for $|z| \leq 1-2^{-(2 n-1)}$;

(v) $\left|g_{n}(z)\right| \leq n^{4} 2^{2 n+1}$ for $z \notin \bigcup_{\Delta \in A(n)} \Delta$;

(vi) $g_{n}(z) \neq 0$ for all $z$;

(vii) $\bigcup_{\Delta \in A(n)} \Delta \subset\left\{z: 2^{-(2 n-1)} \leq|z| \leq 1-2^{-(2 n+1)}\right\}$.

Proof. Let $N=n 2^{2 n}, \omega=\exp (2 \pi / N), g_{n}=h_{N}\left(\omega^{-1 / 2}\left(1-2^{-2 n}\right)^{-1} z\right)$. If we take $A(n)$ to be the collection of discs with radii $n^{-5} 2^{-4 n}$ and centres 
$\left(1-2^{-2 n}\right) \omega^{r+1 / 2}(0 \leq r \leq N-1)$, results (ii)-(vii) are either trivial or follow from Lemma 2.3 on scaling by a factor of $\omega^{1 / 2}\left(1-2^{-2 n}\right)$.

To show part (i), consider first those discs with centres $\left(1-2^{-2 n}\right) \omega^{r+1 / 2}$ $(0 \leq r \leq(N-1) / 8)$. For such a disc $\Delta$ we have

$$
\begin{aligned}
s_{0}(\Delta) & =\left(1-2^{-2 n}\right) \sin \left(\frac{(r+1 / 2) \pi}{n 2^{2 n}}\right)-n^{-5} 2^{-4 n} \\
& \geq \frac{(r+1 / 2) \pi}{n 2^{2 n+2}}-n^{-5} 2^{-4 n} \geq\left(\frac{1}{2}\right) \frac{2 r+1}{n 2^{2 n}} .
\end{aligned}
$$

So

$$
\frac{r(\Delta)}{s_{0}(\Delta)^{2}} \leq n^{-5} 2^{-4 n} \frac{4 n^{2} 2^{4 n}}{(2 r+1)^{2}}=\frac{4 n^{-3}}{(2 r+1)^{2}}
$$

and

$$
\sum_{k=1}^{N / 8-1} \frac{r\left(\Delta_{k}\right)}{s_{0}\left(\Delta_{k}\right)} \leq 4 n^{-3} \sum_{k=0}^{N / 8-1}(2 k+1)^{-2} \leq 4 n^{-3} \sum_{k=0}^{\infty} k^{-2} \leq \frac{n^{-2}}{8}
$$

provided only that $n>K=32 \sum_{r=0}^{\infty} r^{-2}$. So, by symmetry,

$$
\sum_{\Delta \in A(n)} \frac{r(\Delta)}{s_{0}(\Delta)^{2}} \leq n^{-2}
$$

provided only that $n$ is sufficiently large.

Multiplying the $g_{n}$ together as in [7], we obtain the following.

Lemma 2.5. Given any $\varepsilon>0$ there exists an $m=m(\varepsilon)$ such that if (adopting the notation of Lemma 2.4) we let $f_{n}=(m !)^{-4} \prod_{r=m}^{n} g_{r}$ and $\left\{\Delta_{k}\right\}$ be a sequence enumerating the discs of $\bigcup_{r=m}^{\infty} A(r)$ then the following hold:

(a) $\sum_{k=1}^{\infty} r\left(\Delta_{k}\right) / s_{0}\left(\Delta_{k}\right)^{2}<\varepsilon$ and so then $\sum_{k=1}^{\infty} r\left(\Delta_{k}\right)<\varepsilon$;

(b) the poles of the $f_{n}$ lie in $\bigcup_{k=1}^{\infty} \Delta_{k}$;

(c) the sequence $\left\{f_{n}\right\}$ tends uniformly to zero on $\{z \in \mathbb{C}:|z| \geq 1\}$.

Proof. Observe that

$$
\sum_{k=1}^{\infty} r\left(\Delta_{k}\right) \leq \sum_{k=1}^{\infty} \frac{r\left(\Delta_{k}\right)}{s_{0}\left(\Delta_{k}\right)^{2}}=\sum_{l=m}^{\infty} \sum_{\Delta \in A(l)} \frac{r(\Delta)}{s_{0}(\Delta)^{2}} \leq \sum_{l=m}^{\infty} l^{-2}<\varepsilon
$$

provided only that $m(\varepsilon)>2 \varepsilon^{-1}+1$. Thus conclusions (a) and (b) are easy to verify. To prove (c), set $K=\prod_{r=1}^{\infty}\left(1+(r+1)^{-4}\right)$ and observe that, provided $m(\varepsilon)$ is large enough that $(m+1) !^{4}>2(m+1)^{6} 2^{2(m+1)}+2(m+1)^{2}$, if we let $z \notin \bigcup_{k=1}^{\infty} \Delta_{k}$, a simple induction gives 
$\left|f_{n}(z)\right| \leq \begin{cases}(n+1) !^{-4} & \text { for } 1-2^{-(2 n+1)}<|z|, \\ n^{-2} \leq \prod_{r=m}^{n}\left(1+(r+1)^{-4}\right) \leq K & \text { for } 1-2^{-(2 n-1)}<|z|<1-2^{-(2 n+1)}, \\ \prod_{r=m}^{n}\left(1+(r+1)^{-4}\right) \leq K & \text { for }|z|<1-2^{-(2 n-1)} .\end{cases}$

Using the trivial equality

$$
\left|f_{n+1}(z)-f_{n}(z)\right|=\left|f_{n}(z)\right|\left|1-g_{n+1}(z)\right|,
$$

we see that for $z \notin \bigcup_{k=1}^{\infty} \Delta_{k}$,

$$
\left|f_{n+1}(z)-f_{n}(z)\right| \leq \begin{cases}K(n+1)^{-4} & \text { for }|z| \leq 1-2^{-(2 n+1)}, \\ (n+1) !^{-4}\left(1+(n+1)^{4} 2^{2 n+1}\right) \leq(n+1)^{-2} & \text { for } 1-2^{-(2 n+1)}<|z| .\end{cases}
$$

Thus $\left|f_{n+1}(z)-f_{n}(z)\right| \leq K(n+1)^{-2}$ for all $z \notin \bigcup_{k=1}^{\infty} \Delta_{k}$ and, by for example the Weierstrass $M$ test, $f_{n}$ converges uniformly to $f$ say. To see that $f(z) \neq 0$ for $|z|<1, z \notin \bigcup_{k=1}^{\infty} \Delta_{k}$, note that if $|z| \leq 1-2^{2 n-1}$ then $f_{n}(z) \neq 0$, and

$$
\sum_{r=n+1}^{\infty}\left|1-g_{r}(z)\right| \leq \sum_{r-n+1}^{\infty}(r+1)^{-4}<\infty .
$$

So by a basic result on infinite products (see, for example, $[10,15.5]$ ),

$$
f(z)=f_{n}(z) \prod_{r=n+1}^{\infty} g_{r}(z) \neq 0 .
$$

Hence by dilation and translation we obtain the following.

Lemma 2.6. Given any closed disc D, with centre a and radius $r$, and any $\varepsilon>0$, we can find a sequence $\left\{\Delta_{k}\right\}$ of open discs and a sequence $\left\{f_{n}\right\}$ of rational functions such that, letting $s_{1}(\Delta):=\operatorname{dist}\left(\Delta_{k}, a+\mathbb{R} \cup i \mathbb{R}\right)$ for $a$ disc $\Delta$, the following hold:

(a) $\sum_{k=1}^{\infty} r\left(\Delta_{k}\right) / s_{1}\left(\Delta_{k}\right)^{2}<\varepsilon$ and so $\sum_{k=1}^{\infty} R\left(\Delta_{k}\right)<\varepsilon$;

(b) the poles of the $f_{n}$ lie in $\bigcup_{k=1}^{\infty} \Delta_{k}$;

(c) the sequence $\left\{f_{n}\right\}$ tends uniformly to zero on $(\mathbb{C} \backslash D) \backslash \bigcup_{k=1}^{\infty} \Delta_{k}$.

We are now ready to prove Theorem 2.1.

Proof of Theorem 2.1. Let $\left\{D_{l}\right\}_{l=1}^{\infty}$ be an enumeration of all closed discs of centre $z$ and radius $r$ with $z \in \mathbb{Q}+i \mathbb{Q}$ and $r \in \mathbb{Q}^{+}$such that, letting $K=\{-1-i,-1+i, 1-i, 1+i\}$, one of the following holds:

(1) $z \in \operatorname{int}(Q)$ and $r<\operatorname{dist}(z, \partial Y)$;

(2) $z \in \partial Q \backslash K$ and $r<\operatorname{dist}(z, K)$;

(3) $z \in K$ and $r<1$. 
We apply Lemma 2.6 with $D=D_{l}$ and $\varepsilon=\varepsilon_{l}$ where

$$
\varepsilon_{l}< \begin{cases}2^{-l-1} C_{0} \operatorname{dist}\left(D_{l}, \partial Q\right)^{2} & \text { if } D_{l} \text { is of type (1), } \\ 2^{-l-1} C_{0} \operatorname{dist}\left(D_{l}, K\right)^{2} & \text { if } D_{l} \text { is of type (2), } \\ 2^{-l-1} C_{0} & \text { if } D_{l} \text { is of type (3), }\end{cases}
$$

to obtain $\left(\Delta_{l, n}\right)$ and $\left(f_{l, k}\right)$. Let $\left\{U_{N}\right\}_{N=1}^{\infty}$ be a sequence enumerating the $\Delta_{n, a}$ and

$$
X_{0}=Q \backslash \bigcup_{N=1}^{\infty} U_{N}
$$

We have

$$
\sum_{N=1}^{\infty} \frac{r\left(U_{N}\right)}{s\left(U_{N}\right)^{2}}=\sum_{l=1}^{\infty} \sum_{n=1}^{\infty} \frac{r\left(\Delta_{l, n}\right)}{s\left(\Delta_{l, n}\right)^{2}}<\sum_{l=1}^{\infty} C_{0} 2^{-l}=C_{0} .
$$

Given any point $z$ in $X_{0}$ and any compact set $B \subset X_{0}$ there exists $D_{l}$ with $z \in D_{l}$ and $B \cap D_{l}=\emptyset$. Hence $f_{l}:=\lim _{k \rightarrow \infty} f_{l, k} \in R\left(X_{0}\right)$ has $f_{l}(z) \neq 0$ and $f_{l}(B) \subset\{0\}$, so $R\left(X_{0}\right)$ is regular.

In order to prove Theorem 1.1 we need some further lemmas. The first one is trivial.

Lemma 2.7. Let $X, Y$ be compact plane sets with $X \subset Y$. If $R(Y)$ is regular, then the same is true for $X$.

The next two results are essentially the same as those used in [6].

Lemma 2.8. Let $\left(\chi_{n}\right)$ be a sequence of Jordan domains whose closures are contained in $Q$. Set $X_{2}=Q \backslash \bigcup_{n=1}^{\infty} \chi_{n}$. Let $s_{n}$ be the distance from $\chi_{n}$ to $\partial Q$ and let $c_{n}$ be the length of the boundary of $\chi_{n}$. Let $f$ and $g$ be in $R_{0}\left(X_{2}\right)$. Then

$$
\left|\int_{\partial Q} f^{\prime}(z) g(z) d z\right| \leq 2|f|_{X}|g|_{X} \sum_{n=1}^{\infty} \frac{c_{n}}{s_{n}^{2}}
$$

Proof. The argument of $[6,2.1]$ applies.

Lemma 2.9. Let $X$ be a compact subset of $Q$. Suppose that there is a sequence of real numbers $L_{n} \in(0,1)$ such that $L_{n} \rightarrow 1$ and, for each $n$, $R\left(X \cap L_{n} Q\right)$ has no non-zero bounded point derivations. Then $R(X)$ has no non-zero bounded point derivations.

Proof. The argument of $[6,2.2]$ applies.

The following is $[6,2.3]$.

Lemma 2.10. Let $X, Y$ be compact plane sets with $X \subset Y$. If $R(Y)$ has no non-zero, bounded point derivations, then the same is true for $X$.

The following result was proved by Wermer [12]. 
Proposition 2.11. Let $D$ be a closed disc in $\mathbb{C}$ and let $\varepsilon>0$. Then there is a sequence of open discs $U_{k} \subset D$ such that $R\left(D \backslash \bigcup_{k=1}^{\infty} U_{k}\right)$ has no non-zero bounded point derivations but the sum of the radii of the discs $U_{k}$ is less than $\varepsilon$.

Corollary 2.12. Let $Y$ be a square set of the form $r Q+z$ and $\delta>0$. Then there is a sequence of Jordan domains $\chi_{l} \subset Y$ such that $R\left(Y \backslash \bigcup_{l=1}^{\infty} \chi_{l}\right)$ has no non-zero bounded point derivations but the sum of the lengths of the boundaries of the $\chi_{l}$ is less than $\delta$.

Proof. Apply the previous proposition to any closed disc containing $Y$ with $\varepsilon<\delta /(2 \pi)$. Then, by Lemma 2.10 , letting $\left(\chi_{l}\right)$ be a sequence enumerating all non-empty sets of the form $U_{k} \cap \operatorname{int}(Y)$ will suffice.

Proof of Theorem 1.1. Let $C>0$. Set $L_{n}=n /(n+1)$. Applying Corollary 2.12 to $L_{n} Q$ we may choose Jordan domains $\chi_{n, k} \subset L_{n} Q$ such that $R\left(L_{n} Q \backslash \bigcup_{k=1}^{\infty} \chi_{n, k}\right)$ has no non-zero bounded point derivations and the sum of the lengths of the boundaries of the $\left(\chi_{n, k}\right)_{k=0}^{\infty}$ is less than $2^{-(n+1)} C\left(1-L_{n}\right)^{2}$. Set

$$
X_{2}=Q \backslash \bigcup_{n, k} \chi_{n, k}
$$

and let

$$
X_{1}=Q \backslash \bigcup_{n} D_{n}
$$

be the result of applying Theorem 2.1 with $C_{0}=C /(4 \pi)$. Finally set $X=X_{1} \cap X_{2}$. Then Lemma 2.7 gives that $R(X)$ is regular and Lemmas 2.9 and 2.10 give that $R(X)$ has no non-zero bounded point derivations. Enumerating the sets $\chi_{n, k}$ and $D_{n}$ as $C_{1}, C_{2}, \ldots$ we may apply Lemma 2.8 to obtain the required estimate on the integral.

3. Regularity and peak points. A point $x$ in the character space $X$ of a uniform algebra $A$ is said to be a peak point for $A$ if there is $f \in A$ such that $f(x)=1$ and $|f(y)|<1$ for all $y \in X \backslash\{x\}$; and $x$ is a point of continuity for $A$ if, for every compact set $K \subset X \backslash\{x\}$, there is a function $f$ in $A$ such $f(x)=1$ and $f(K) \subset\{0\}$. Notice that a uniform algebra is regular if and only if every point in its character space is a point of continuity. The following result, regarding systems of Cole root extensions (see [2]), is [5, $2.8]$.

Proposition 3.1. Let $A$ and $B$ be uniform algebras such that $B$ is the result of applying a system of Cole root extensions to $A$. If $A$ is regular so is $B$.

In [6] an example of a separable uniform algebra, $A$, such that every point of the character space is a peak point for $A$ and $A$ is not weakly amenable 
is obtained by first modifying the original example so that every point, except possibly those on the outer boundary circle, is a point of continuity, and then applying an appropriate system of Cole root extensions. Following essentially the same argument and noting the above proposition we obtain the following.

THEOREM 3.2. There exists a regular uniform algebra $A$ whose character space is metrizable such that every point of the character space of $A$ is a peak point but $A$ is not weakly amenable.

We note that both this algebra and the algebra constructed in Theorem 1.1 have dense invertible group (by results in [4]).

We finish by noting that we do not know whether or not either of the uniform algebras we have constructed are strongly regular - i.e. if, for each point $x$ in the character space, the algebra of functions constant on a neighbourhood of $x$ is dense in the original algebra.

\section{References}

[1] W. G. Bade, P. C. Curtis and H. G. Dales, Amenability and weak amenability for Beurling and Lipschitz algebras, Proc. London Math. Soc. (3) 55 (1987), 359-377.

[2] B. J. Cole, One point parts and the peak point conjecture, Ph.D. Thesis, Yale Univ., 1968.

[3] H. G. Dales, Banach Algebras and Automatic Continuity, Oxford Univ. Press, New York, 2000.

[4] T. W. Dawson and J. F. Feinstein, On the denseness of the invertible group in Banach algebras, Proc. Amer. Math. Soc. 131 (2003), 2831-2839.

[5] J. F. Feinstein, A non-trivial, strongly regular uniform algebra, J. London Math. Soc. 45 (1992), 288-300.

[6] -, A counterexample to a conjecture of S. E. Morris, Proc. Amer. Math. Soc. 132 (2004), 2389-2397.

[7] T. W. Körner, A cheaper Swiss cheese, Studia Math. 83 (1986), 33-36.

[8] R. McKissick, A nontrivial normal supnorm algebra, Bull. Amer. Math. Soc. 69 (1963), 391-395.

[9] S. E. Morris, Bounded derivations from uniform algebras, Ph.D. thesis, Univ. of Cambridge, 1993.

[10] W. Rudin, Real and Complex Analysis, 3rd ed., McGraw-Hill, Singapore, 1987.

[11] E. L. Stout, The Theory of Uniform Algebras, Bogden and Quigley, New York, 1971.

[12] J. Wermer, Bounded point derivations on certain Banach algebras, J. Funct. Anal. 1 (1967), 28-36.

School of Mathematical Sciences

University of Nottingham

Nottingham NG7 2RD, England

E-mail: pmxmjh@nottingham.ac.uk 